\title{
Efficacy and tolerability of a house dust mite allergoid in allergic bronchial asthma: a randomized dose-ranging trial
}

\author{
Marek Jutel*,1,2, Michael Rudert ${ }^{3}$, Fabian Kreimendahl ${ }^{3}$ \& Piotr Kuna ${ }^{4}$ \\ 1 "ALL-MED" Medical Research Institute, Wroclaw, Poland \\ ${ }^{2}$ Department of Clinical Immunology, Silesian Piasts University of Medicine, Wroclaw, Poland \\ ${ }^{3}$ Allergopharma GmbH \& Co. KG, Medical Department, Reinbek, Germany \\ ${ }^{4}$ Barlicki University Hospital, Medical University, Lodz, Poland \\ *Author for correspondence: Tel.: +48 71 3633356; Fax: +48 71 3633355; marek.jutel@all-med.wroclaw.pl
}

\begin{abstract}
Aim: This multicenter randomized placebo-controlled double-blind clinical trial investigated which maintenance dose shows the optimal benefit-risk ratio for subcutaneous immunotherapy with a Dermatophagoides pteronyssinus allergoid preparation. Objective: To evaluate four maintenance doses of the allergoid preparation versus placebo. Methods: The late-phase reaction of the intracutaneous test was chosen as efficacy parameter and minimal dose of fluticasone required for asthma control. Results: A total of 146 adults with bronchial asthma were randomized. After subcutaneous immunotherapy, reductions in swelling size were greatest with 10,000 therapeutic units (TU). The 18,000 TU group showed the highest percentage of patients with fluticasone dose reduced to $0 \mu \mathrm{g} /$ day. Conclusion: The optimal dose of allergoid for the investigation in a confirmatory trial with inhaled corticosteroid reduction is 18,000 TU.
\end{abstract}

First draft submitted: 28 June 2018; Accepted for publication: 18 July 2018; Published online: 21 September 2018

Keywords: allergen immunotherapy $\bullet$ allergic bronchial asthma $\bullet$ allergoid $\bullet$ house dust mite $\bullet$ randomized doseranging trial • rhinitis $\bullet$ rhinoconjunctivitis $\bullet$ subcutaneous immunotherapy

House dust mite (HDM) allergy is the most prevalent IgE-mediated indoor allergy and may be associated with bronchial asthma, rhinitis and/or rhinoconjunctivitis [1,2]. Allergen immunotherapy (AIT) is the only causative treatment for IgE-mediated allergy [2,3]. Subcutaneous allergen immunotherapy (SCIT) is the most common form of AIT used for this type of allergy worldwide.

Application of native allergens for AIT dates to over a century. Chemical modification of allergens was introduced to increase the safety profile of AIT by substantially reducing allergenicity, as judged by skin prick testing, provocation testing, histamine release from sensitized leukocytes and measurement of IgE-binding activity [4-7]. Immunogenic activity and T-cell reactivity are retained [7]. These features account for a reduced risk of IgE-mediated side reactions and the possibility of administration of higher doses of immunogen over a shorter time compared with native allergens.

The SCIT allergoid preparation under investigation is a modified formulation of the HDM Dermatophagoides pteronyssinus. For details of the production of this and other SCIT allergoid preparations, see the Supplementary Material and previous publications $[4,8]$.

The efficacy and safety of SCIT with this allergoid preparation have been investigated in HDM-induced bronchial asthma, showing promising first results. The minimum inhaled corticosteroid (ICS) dose required for asthma control was significantly reduced during AIT-SCIT treatment with 6000 therapeutic units (TU) versus placebo in 65 pediatric patients [9].

The aim of the current Phase II trial was to determine the optimal dose for efficacy and tolerability of SCIT with the $D$. pteronyssinus allergoid preparation in adults with well-controlled allergic bronchial asthma and allergic rhinitis (AR) or allergic rhinoconjunctivitis induced by HDM. The results will serve the optimization of the following confirmatory trial. The trial also investigated whether the D. pteronyssinus SCIT preparation is able to induce Dermatophagoides farinae-specific $\operatorname{IgG}$ and $\operatorname{IgG} 4$ antibody responses. 
Considering the positive outcome of previous studies, the change of the weal size in intracutaneous test (ICT) measured after $6 \mathrm{~h}$ (late phase) assessed before and after AIT treatment was chosen as the primary end point in this study [10-12].

\section{Methods}

Trial design

This multicenter, randomized, double-blind and placebo-controlled dose-ranging trial with parallel groups at different doses of SCIT were conducted in 32 centers in Poland and six centers in Spain between April 2012 and April 2015.

\section{Objective}

To evaluate the efficacy and safety of four different maintenance doses $(2000,6000,10,000$ and 18,000 TU) of D. pteronyssinus allergoid preparation (Acaroid ${ }^{\circledR}$, Allergopharma GmbH \& Co. KG, Reinbek, Germany) in adults with controlled allergic bronchial asthma and AR or allergic rhinoconjunctivitis caused by HDM.

\section{Trial population}

For inclusion, the following criteria were applied: patients aged 18-40 years with allergic bronchial asthma caused by HDM sensitization documented by skin prick test wheal for $D$. pteronyssinus $\geq 3 \mathrm{~mm}$ in diameter; immunoassay result for $D$. pteronyssinus-specific $\mathrm{IgE}>1.5 \mathrm{kU} / \mathrm{l}$; proven clinical relevance of $\mathrm{HDM}$ allergy by positive conjunctival provocation test with $D$. pteronyssinus allergens; peak expiratory flow (PEF) or forced expiratory volume in $1 \mathrm{~s}$ $\left(\mathrm{FEV}_{1}\right) \geq 80 \%$ of predicted normal.

Patients selection were based on: a confirmed asthma diagnosis, classified as 'controlled' according to GINA guidelines [13]; a history of ICS treatment with a fluticasone equivalent of up to a maximum dose of $500 \mu \mathrm{g} / \mathrm{day}$; late-phase reaction (LPR) in ICT at baseline $\geq 20 \mathrm{~mm}$ in longest diameter.

\section{Procedures}

In this dose range finding (DRF) phase, a 1:1:1:1:1 randomization ratio for the four doses of SCIT and placebo were used. Randomization was performed independent centrally. The treatment schedule comprised of subcutaneous injections of Acaroid ${ }^{\circledR}$ or placebo, administered for approximately 7 months during the DRF phase, which was divided into a dose-escalation phase and a dose-maintenance phase. Patients and investigators were blinded to treatment. The randomization, dosing and assessment schedules are described in more detail in the Supplementary Material, including in Supplementary Tables $1 \& 2$ and Supplementary Figure 1.

ICT solution was always applied on the dorsal side of the forearm. The skin reaction was assessed using an optical device (Primos 3D, GF Messtechnik GmbH, Teltow, Germany) to objectively measure the wheal swelling area (LPR) and volume induced by the ICT solution of $D$. pteronyssinus allergen extracts of 5000 standardized biological unit per $\mathrm{ml}(\mathrm{SBE} / \mathrm{ml})$ of $D$. pteronyssinus [10]. The camera provides a measurement with a height resolution of $>20 \mu \mathrm{m}$. Within the gauge field of $160 \times 100 \times 90 \mathrm{~mm}$ the area and volume of the skin reaction can be calculated at inevitable time points [14].

In addition to measuring of the wheal size after ICT, efficacy was assessed by investigating the effects of SCIT on asthma control (using the Asthma Control Test $[\mathrm{ACT}]$ ), lung function (morning PEF) and the serum concentrations of antibodies (IgE, IgG and $\mathrm{IgG}_{4}$ specific to HDMs using ImmunoCAP [ThermoFisher Scientific, Uppsala, Sweden]) at screening and after SCIT. The ACT is a validated five-item patient questionnaire used to determine whether asthma symptoms are well controlled during the previous 4 weeks. Higher scores indicate better asthma control. During screening, the dose of fluticasone was reduced in predefined steps from $2 \times 250 \mu \mathrm{g} / \mathrm{day}$ to $2 \times 100 \mu \mathrm{g} /$ day and $0 \mu \mathrm{g} /$ day. The ACT was undertaken at each step. Each dose-step lasted for 6 weeks until uncontrolled conditions were reached and patients with controlled asthma without fluticasone were excluded from the ACT analyses. After SCIT, patients received the fluticasone dose at which their asthma was uncontrolled at baseline and subsequently undertook the ACT.

\section{Outcomes}

The primary efficacy end point was the absolute change of the swelling area of the LPR after ICT from baseline to after the course of SCIT. 
Secondary end points were changes in the following variables from baseline to after SCIT: swelling area and volume 20 min (early-phase reaction [EPR]) after ICT; minimal ICS dose for asthma control; ACT score; morning PEF; concentrations of $\operatorname{IgE}, \operatorname{IgG}$, and $\mathrm{IgG}_{4}$ specific to D. pteronyssinus and $D$. farinae.

The following safety measures were assessed: treatment-emergent adverse events (TEAEs), safety laboratory, vital signs, and lung function tests at screening and at the end of the trial. Systemic AEs were graded according to the WAO system [15].

\section{Statistical methods}

Primary and secondary efficacy end points were analyzed by analysis of covariance, with change of the parameter as the dependent variable, treatment and center as fixed effects and the baseline value of the parameter as covariable. Change of minimal ICT dose for asthma control was summarized as a continuous variable and in shift tables. Change in $\mu \mathrm{g} /$ day and in dose steps were analyzed with the Mann-Whitney $U$ and Mantel-Haenszel tests, respectively. Fisher's exact test was performed for categorical variables. p-values $<0.05$ were considered statistically significant.

The dose-response relationship of the four doses studied is performed in an exploratory manner and thus, no formal hypotheses are tested. It is assumed that 32 patients per treatment group will be sufficient to get valid information about the dose-response curve of the investigational treatment. Thus, it is planned, that 300 patients will be screened to randomize 160 patients to the five treatment groups.

All efficacy analyses were conducted using the full analysis set (FAS; patients who received $\geq 1$ injection of trial medication or placebo and for whom an efficacy assessment was available) and per-protocol set (PPS; patients of the FAS without major protocol violations). Data for per-protocol set are not shown in this report. All safety analyses were conducted with patients who received $\geq 1$ injection of trial medication or placebo.

Additional statistical methods are in the Supplementary Material.

\section{Results}

A total of 146 patients were randomized to the four active treatment groups and one placebo group (Figure 1); $91.1 \%$ of the 146 patients completed the trial ( $90.6 \%$ in the placebo group vs $83.9-100 \%$ in the active groups).

Overall, the five groups were comparable with respect to demographic characteristics. As shown in Table 1, the mean \pm SD age of the overall population was $27.5 \pm 6.8$ years. Almost half $(47.9 \%)$ of the patients were female. Most groups were well balanced by sex, although women comprised 35.5 and $65.6 \%$ of the $18,000 \mathrm{TU}$ and placebo groups, respectively (Table 1). Mean \pm SD BMI was $24.46 \pm 4.42 \mathrm{~kg} / \mathrm{m}^{2}$. Most patients were Caucasian (98.6\%), had no pets $(78.8 \%)$ and only $1.4 \%(\mathrm{n}=2)$ were smokers.

All patients had HDM allergic asthma and rhinitis symptoms (Table 1), which were present the whole year in 91.8 and $84.2 \%$ of patients, respectively. Most patients had conjunctivitis allergica (70.5\%), whereas few patients (7.5\%) reported atopic dermatitis; the five groups were well balanced with regard to allergic symptoms (Table 1). $11.6 \%(n=17)$ of patients reported other allergic diseases, including allergies to animals and pollen.

While there was some variation between the groups regarding the dose of fluticasone needed to achieve asthma control at baseline, there were no statistically significant differences between the active groups versus the placebo group (Table 1). The 18,000 TU dose group tended to have higher total and HDM-specific IgE concentrations versus the placebo and other dose groups (Table 1). All patients had PEF or $\mathrm{FEV}_{1}>80 \%$ of predicted normal (Table 1). All treatment groups were similar with respect to reactions to the different substances in the SPTs, including D. pteronyssinus and D. farinae (Supplementary Table 3).

\section{Changes in wheal size}

As shown in Figure 2 and Table 2, after SCIT there were statistically significant reductions in the swelling area of the LPR in all four-dose groups. Reductions were also seen in the EPR swelling area (secondary end point), with a statistically significant effect compared with placebo present for the 10,000 TU group (Table 2). The greatest reductions in the least square (LS) means of the swelling area were found in the $10,000 \mathrm{TU}$ group (LPR $-10.83 \mathrm{~cm}^{2}$; EPR $-3.01 \mathrm{~cm}^{2}$ ) versus placebo (LPR $+6.41 \mathrm{~cm}^{2}, \mathrm{p}<0.0001$; EPR $\left.-0.79 \mathrm{~cm}^{2}, \mathrm{p}=0.0063\right)$.

\section{Changes in asthma control}

Over half $(53.7 \%, \mathrm{n}=73)$ of the patients in the FAS already had asthma control without ICS before receiving SCIT and thus were not analyzed for asthma control end points. 
Table 1. Patient characteristics at baseline in the dose range-finding phase - safety set.

\begin{tabular}{|c|c|c|c|c|c|c|}
\hline Baseline characteristics & Placebo $(n=32)$ & $2000 \mathrm{TU}(\mathrm{n}=24)$ & $6000 \mathrm{TU}(\mathrm{n}=31)$ & $10,000 \mathrm{TU}(\mathrm{n}=28)$ & 18,000 TU $(n=31)$ & Overall $(n=146)$ \\
\hline \multicolumn{7}{|l|}{ Age, years: } \\
\hline - Mean (SD) & $28.3(7.5)$ & $25.4(5.0)$ & $27.5(7.3)$ & $27.9(6.7)$ & $28.0(7.0)$ & $27.5(6.8)$ \\
\hline -Min-Max & $18-40$ & $18-40$ & $18-40$ & $18-39$ & $18-40$ & $18-40$ \\
\hline \multicolumn{7}{|l|}{ Sex, $\mathrm{n}(\%)^{\dagger}:$} \\
\hline - Female & $21(65.6)$ & $12(50.0)$ & $14(45.2)$ & $12(42.9)$ & $11(35.5 \%)$ & $70(47.9)$ \\
\hline - Male & $11(34.4)$ & $12(50.0)$ & $17(54.8)$ & $16(57.1)$ & $20(64.5 \%)$ & $76(52.1)$ \\
\hline \multicolumn{7}{|l|}{ Household pets, n (\%): } \\
\hline - At present & $5(15.6)$ & $3(12.5)$ & $6(19.4)$ & $3(10.7)$ & $3(9.7 \%)$ & $20(13.7)$ \\
\hline - Formerly & $3(9.4)$ & $2(8.3)$ & $3(9.7)$ & $1(3.6)$ & $2(6.5 \%)$ & $11(7.5)$ \\
\hline - No pets & $24(75.0)$ & $19(79.2)$ & $22(71.0)$ & $24(85.7)$ & $26(83.9 \%)$ & $115(78.8)$ \\
\hline \multicolumn{7}{|c|}{ History of allergy symptoms, n (\%): } \\
\hline - Allergic asthma & $32(100.0)$ & $24(100.0)$ & $31(100.0)$ & $28(100.0)$ & $31(100.0)$ & $146(100.0)$ \\
\hline - Rhinitis allergica & $32(100.0)$ & $24(100.0)$ & $31(100.0)$ & $28(100.0)$ & $31(100.0)$ & $146(100.0)$ \\
\hline - Cough/sibilant rhonchi ${ }^{\dagger}$ & $24(75.0)$ & $19(79.2)$ & $24(77.4)$ & $19(67.9)$ & $26(83.9)$ & $112(76.7)$ \\
\hline - Conjunctivitis allergica ${ }^{\dagger}$ & $25(78.1)$ & $16(66.7)$ & $23(74.2)$ & $21(75.0)$ & $18(58.1)$ & $103(70.5)$ \\
\hline - Sneezing ${ }^{\dagger}$ & $21(65.6)$ & $17(70.8)$ & $22(71.0)$ & $19(67.9)$ & $24(77.4)$ & $103(70.5)$ \\
\hline - Atopic dermatitis ${ }^{\dagger}$ & $3(9.4)$ & $1(4.2)$ & $2(6.5)$ & $1(3.6)$ & $4(12.9)$ & $11(7.5)$ \\
\hline \multicolumn{7}{|c|}{ Fluticasone dose needed for asthma control, $\mathrm{n}(\%)^{\dagger}$ : } \\
\hline$-0 \mu \mathrm{g} /$ day & $17(53.1)$ & $12(50.0)$ & $17(54.8)$ & $19(67.9)$ & $14(45.2)$ & $79(54.1)$ \\
\hline$-200 \mu \mathrm{g} /$ day & $3(9.4)$ & $7(29.2)$ & $8(25.8)$ & $5(17.9)$ & $5(16.1)$ & $28(19.2)$ \\
\hline$-500 \mu \mathrm{g} /$ day & $12(37.5)$ & $5(20.8)$ & $6(19.4)$ & $4(14.3)$ & $12(38.7)$ & $39(26.7)$ \\
\hline \multicolumn{7}{|l|}{ Mean (SD) IgE, IU/ml‡: } \\
\hline - D. pteronyssinus & $21.1(24.7)$ & $22.79(20.37)$ & $28.8(29.4)$ & $20.7(22.4)$ & $33.4(28.0)$ & $25.6(25.7)$ \\
\hline -D. farinae & $19.6(22.6)$ & $20.8(18.6)$ & $27.8(26.4)$ & $16.2(17.9)$ & $32.2(29.1)$ & $23.6(24.1)$ \\
\hline - Total & $184.4(195.5)$ & $209.8(168.8)$ & $319.4(459.4)$ & $199.3(163.6)$ & $306.9(417.9)$ & $246.4(318.4)$ \\
\hline \multicolumn{7}{|c|}{$\mathrm{FEV}_{1}$ in relation to predicted value, $\%$ : } \\
\hline - Mean (SD) $)^{\ddagger}$ & $99.7(10.8)$ & $98.4(9.5)$ & $98.7(10.1)$ & $100.5(11.1)$ & $96.9(8.9)$ & $98.9(10.0)$ \\
\hline$-\mathrm{n}$ (missing) & $18(14)$ & $14(10)$ & $18(13)$ & $15(13)$ & $14(17)$ & $79(67)$ \\
\hline \multicolumn{7}{|c|}{ PEF in relation to predicted value, \%: } \\
\hline - Mean (SD) $)^{\ddagger}$ & $98.1(9.3)$ & $102.4(8.4)$ & $97.7(8.8)$ & $97.4(10.1)$ & $97.0(12.5)$ & $98.3(10.0)$ \\
\hline$-n$ (missing) & $14(18)$ & $10(14)$ & $13(18)$ & $13(15)$ & $17(14)$ & $67(79)$ \\
\hline
\end{tabular}

† When analyzed using Fisher's exact test, comparing active groups versus placebo, no statistically significant differences were found except in sex in the 18,000 TU versus placebo group (two-sided $p=0.0237$ ).

$¥$ When analyzed using Mann-Whitney $U$ test, comparing active groups versus placebo, no statistically significant differences were found except in $D$. pteronyssinus concentration in the 18,000 TU versus placebo group (two-sided $p=0.0398$ ).

D. farinae: Dermatophagoides farinae; D. pteronyssinus: Dermatophagoides pteronyssinus; DRF: Dose range finding; FEV1: Forced expiratory volume in 1 s; Ig: Immunoglobulin; Max: Maximum; Min: Minimum; N: Number of patient in the group; n: Number of patient with data; PEF: Peak expiratory flow; SD: Standard deviation; TU: Therapeutic unit.

\begin{tabular}{|c|c|c|c|c|c|}
\hline $\begin{array}{l}\text { End point (change from } \\
\text { baseline) }\end{array}$ & Placebo $(n=29)$ & $2000 \mathrm{TU}(\mathrm{n}=24)$ & $6000 \mathrm{TU}(\mathrm{n}=30)$ & $10,000 \mathrm{TU}(\mathrm{n}=25)$ & 18,000 TU $(n=28)$ \\
\hline $\operatorname{LPR}\left(\mathrm{cm}^{2}\right)$ & -6.41 & 7.98 & 6.30 & 10.83 & 8.64 \\
\hline $\operatorname{EPR}\left(\mathrm{cm}^{2}\right)$ & 0.79 & 1.15 & 1.99 & 3.01 & 0.91 \\
\hline $\begin{array}{l}\text { ICS* (reduction by two } \\
\text { steps) }\end{array}$ & $20 \%$ & $25 \%$ & $23 \%$ & $25 \%$ & $69 \%$ \\
\hline $\lg _{4} \quad$ D. pter. (mg/l) & -0.03 & -1.07 & -2.44 & -2.16 & -3.04 \\
\hline $\lg G$ D. pter. (mg/l) & -0.54 & -4.02 & -6.47 & -7.56 & -9.38 \\
\hline
\end{tabular}

ACT: Asthma Control Test; D. pter: Dermatophagoides pteronyssinus; EPR: Early-phase reaction; ICS: Inhaled corticosteroid; IgG: Immunoglobulin G; LPR: Late-phase reaction; N: Number of patient with data; PEF: Peak expiratory flow; TU: Therapeutic unit. 


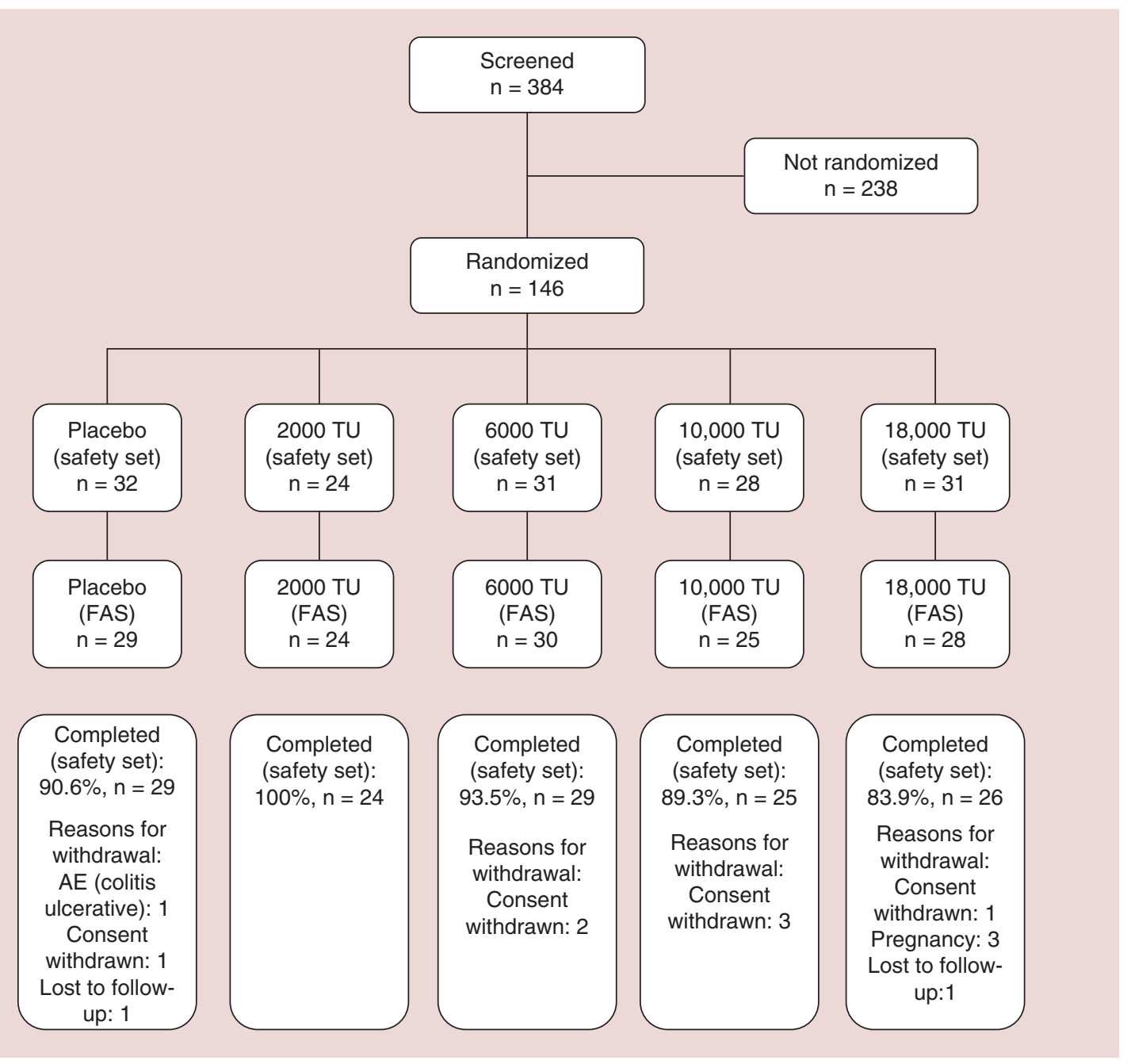

Figure 1. Flow of patients in the dose range finding phase.

AE: Adverse event; DRF: Dose range finding; FAS: Full analysis set; N: Number of patient; TU: Therapeutic unit.

With regard to change in the minimal ICS (fluticasone) dose required for asthma control, the greatest and only statistically significant decrease was in the highest dose group, 18,000 TU, with a mean $\pm S D$ of $-376.9 \pm 200.6 \mu \mathrm{g} / \mathrm{day}$ $(\mathrm{n}=13)$ versus $-100.0 \pm 352.5 \mu \mathrm{g} /$ day with placebo $(\mathrm{n}=15 ; \mathrm{p}=0.0217)$. Similarly, as shown in Table 3 , the percentage of patients who had better asthma control after SCIT, and therefore could reduce their dose of fluticasone, was highest in the $18,000 \mathrm{TU}$ group versus placebo $(69.2 \mathrm{vs} 20.0 \% ; \mathrm{p}=0.0160)$. As shown in Table 3 and Figure 3, 84.6\% of patients reduced their fluticasone dose to $0 \mu \mathrm{g} /$ day in the $18,000 \mathrm{TU}$ group versus $40.0 \%$ in the placebo group $(\mathrm{p}=0.0238)$. However, while average ACT scores increased in all dose groups, the only statistically significant $(\mathrm{p}=0.0432)$ and greatest increase was in the $6000 \mathrm{TU}$ group (LS mean $\pm S E, 4.83 \pm 1.22, \mathrm{n}=9$ ) versus placebo $(1.77 \pm 1.08, \mathrm{n}=13)$.

Average morning PEF increased in all four-dose groups, reaching statistical significance $(\mathrm{p}=0.0165)$ in the 10,000 TU group (LS mean \pm SE, $87.56 \pm 30.99 \mathrm{l} / \mathrm{min}, \mathrm{n}=6)$ versus placebo $(-4.10 \pm 20.90 \mathrm{l} / \mathrm{min}, \mathrm{n}=13)$. The LS means of morning PEF in relation to predicted value (\%) also increased, by $3.51 \%$ in the placebo group, $8.91 \%$ in the 2000 TU group, $7.82 \%$ in the $6000 \mathrm{TU}$ group, $12.71 \%$ in the $10,000 \mathrm{TU}$ group and $4.29 \%$ in the $18,000 \mathrm{TU}$ group, although no increases in the active groups were statistically significant versus placebo.

\section{Changes in the concentrations of HDM-specific antibodies}

As shown in Figure 4 and in Supplementary Table 5, statistically significant increases were detected in the concentrations of $D$. pteronyssinus- and $D$. farinae-specific $\mathrm{IgG}_{4}$ concentrations in the active groups versus placebo, 


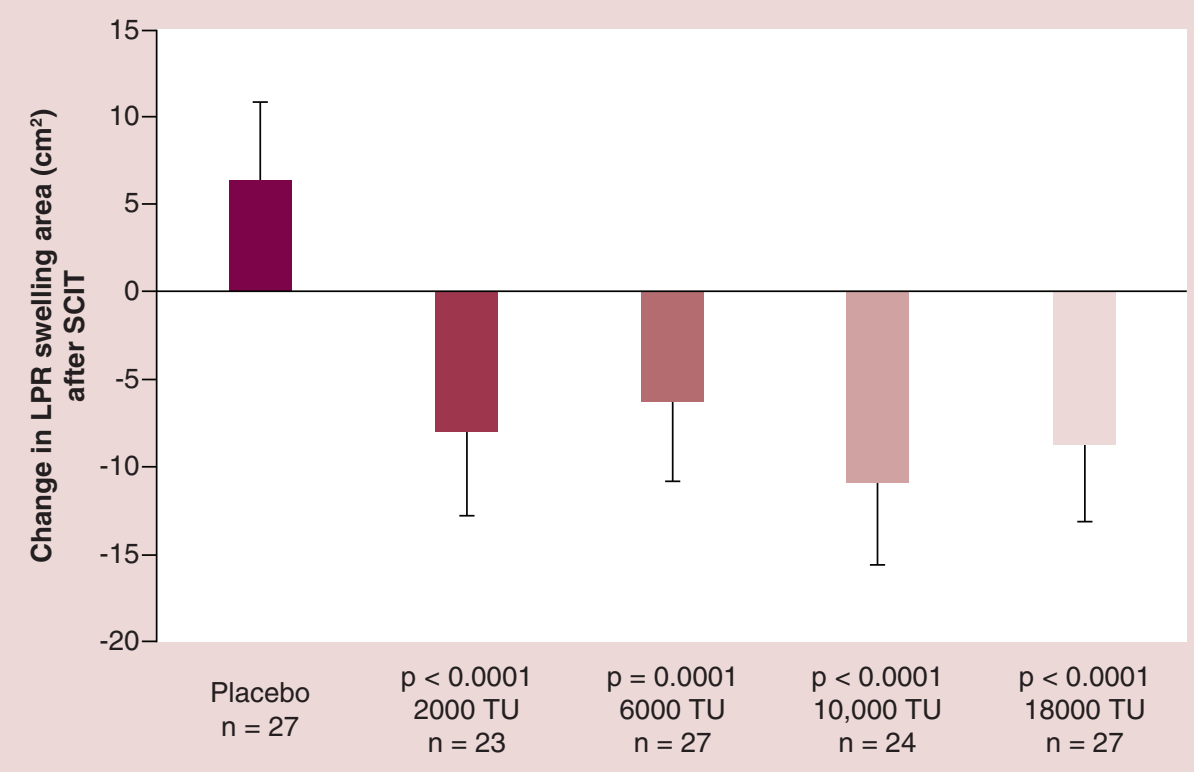

Figure 2. Reduction in swelling area of the late-phase reaction in four dose groups. Changes in swelling area $\left(\mathrm{cm}^{2}\right)$ of the (A) LPR and (B) EPR after SCIT - full analysis set. LPR was $6 \mathrm{~h}$ and EPR was 20 min after injection of the ICT solution. Two-sided analysis of covariance $p$-values were for comparisons between the active dose group and placebo. The bars show the least square means. The whiskers show the upper or lower limits of the $95 \% \mathrm{Cls}$.

LPR: Late-phase reaction; n: Number of patient with data; SCIT: Subcutaneous allergen immunotherapy; TU: Therapeutic unit.

\begin{tabular}{|c|c|c|c|c|c|c|c|c|c|c|}
\hline $\begin{array}{l}\text { Reduction } \\
\text { steps }\end{array}$ & \multicolumn{2}{|c|}{ Placebo $(n=29)$} & \multicolumn{2}{|c|}{$2000 \mathrm{TU}(\mathrm{n}=24)$} & \multicolumn{2}{|c|}{$6000 \mathrm{TU}(\mathrm{n}=30)$} & \multicolumn{2}{|c|}{$10,000 \mathrm{TU}(\mathrm{n}=25)$} & \multicolumn{2}{|c|}{$18,000 \mathrm{TU}(\mathrm{n}=28)$} \\
\hline $\begin{array}{l}\text { Reduction by } \\
\text { two steps } \\
{[-2]}\end{array}$ & $3 / 15$ & $(20.0 \%)$ & $3 / 12$ & $(25.0 \%)$ & $3 / 13$ & $(23.1 \%)$ & $2 / 8$ & $(25.0 \%)$ & $9 / 13$ & $(69.2 \%)$ \\
\hline No change & $3 / 15$ & $(20.0 \%)$ & $3 / 12$ & $(25.0 \%)$ & $2 / 13$ & $(15.4 \%)$ & $1 / 8$ & $(12.5 \%)$ & $2 / 13$ & $(15.4 \%)$ \\
\hline $\begin{array}{l}\text { Increase by } \\
\text { one step } \\
{[+1]}\end{array}$ & $3 / 15$ & $(20.0 \%)$ & $1 / 12$ & $(8.3 \%)$ & $0 / 13$ & $(0.0 \%)$ & $1 / 8$ & $(12.5 \%)$ & $0 / 13$ & $(0.0 \%)$ \\
\hline$p$-value ${ }^{\dagger}$ & & & 0.5693 & & 0.2129 & & 0.6737 & & 0.0160 & \\
\hline
\end{tabular}

Patients who were controlled without fluticasone before treatment and patients who were without any asthma control assessment post-treatment were excluded from the table.

$\dagger p$-value from exact two-sided Mantel-Haenszel $\chi^{2}$ test comparing the active dose group against placebo.

$\ddagger$ p-value from exact two-sided $\chi^{2}$ test comparing the active dose group against placebo.

FAS: Full analysis set; $\mathrm{N}$ : Number of patient in the group; $\mathrm{n} / \mathrm{N}$ : Number of patient with the applicable change of asthma control dose relative to the number of patient with evaluable change of asthma control dose; SCIT: Subcutaneous allergen immunotherapy; TU: Therapeutic unit. 


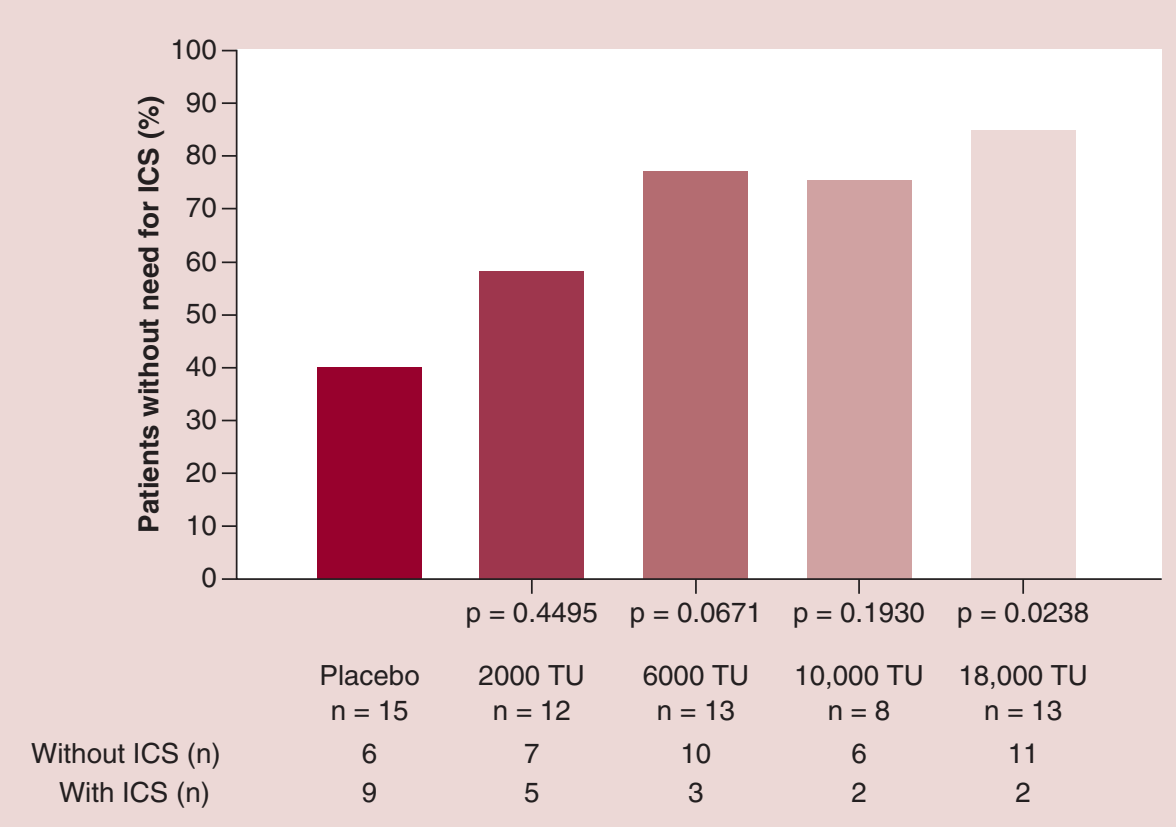

Figure 3. Percentages of patients without need for inhaled corticosteroids after SCIT. Percentages and numbers of patients without need for ICS after subcutaneous allergen immunotherapy - FAS.

$\mathrm{p}$-values are from the exact two-sided $\chi^{2}$ test comparing the active dose group against placebo.

FAS: Full analysis set; ICS: Inhaled corticosteroid; N: Number of patients in the group; $\mathrm{n}$ : Number of patients with data; TU: Therapeutic unit.

D. pteronyssinus- and $D$. farinae-specific IgG concentrations in all four active groups versus placebo. No clinically meaningful changes were detected in $D$. pteronyssinus- and $D$. farinae- specific $\operatorname{IgE}$ concentrations.

\section{Safety}

In total, 178 TEAEs were reported by $48.6 \%(\mathrm{n}=71)$ of patients, including 71 trial medication-related TEAEs reported by $18.5 \%(n=27)$ of patients. Except for local reactions, all doses were comparable to placebo with respect to occurrence of trial medication-related TEAEs (Table 4). More patients in the 18,000 TU dose group $(\mathrm{n}=10)$ experienced trial medication-related local reactions versus the 10,000 TU $(n=4), 6000 \mathrm{TU}(\mathrm{n}=6), 2000 \mathrm{TU}$ $(n=4)$ and placebo groups $(n=0$; Table 4$)$, although all these reactions resolved, were mild or moderate in intensity and were not treatment-limiting.

Only one patient discontinued treatment due to a TEAE (ulcerative colitis; Figure 1). This patient was in the placebo group, and the TEAE was nonserious and not related to trial medication.

Six patients reported a treatment-related systemic reaction, two patients each in the placebo, $6000 \mathrm{TU}$ and 18,000 TU groups: one anaphylactic reaction (WAO grade 2) in the 18,000 TU group and five hypersensitivity reactions (WAO grade 1) including cough, AR and allergic conjunctivitis symptoms. All systemic reactions were mild or moderate in intensity. One adult male patient (aged 30 years) experienced an anaphylactic reaction involving symptoms of urticaria, dyspnea and weakness during the early maintenance phase following the third injection of 18,000 TU. The event was treated with compress-ice pack and oral doses of antihistamine, inhaled $\beta$-agonist and intramuscular corticosteroids and resolved completely the same day. The patient completed the trial with a dose reduction to once $6000 \mathrm{TU}$ and 10,000 TU and three further injections of 18,000 TU without any new local or systemic reaction. This anaphylactic reaction was the only serious TEAE related to trial medication. Five other serious TEAEs, not related to treatment, were reported for four patients (postoperative hernia and appendicitis in the placebo group, asthma exacerbation in the $2000 \mathrm{TU}$ group and varicose vein and umbilical hernia in one patient in the 18,000 TU group).

Laboratory measurements, vital signs and lung function tests did not reveal relevant differences between the different dose and placebo groups (data not shown). 

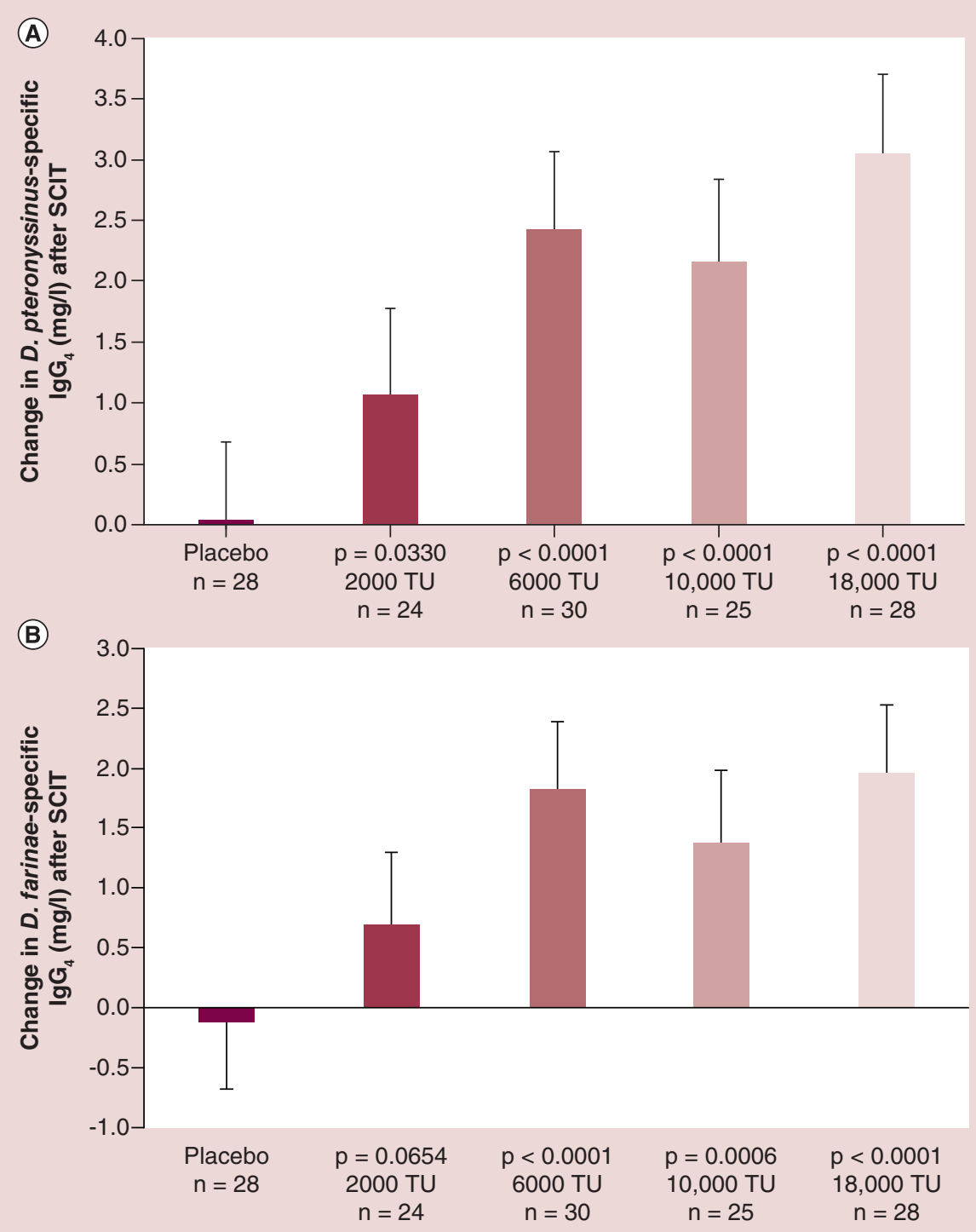

Figure 4. Changes in concentrations of allergen-specific IgG4. Changes in concentrations (mg/l) of IgG 4 specific to (A) D. Pteronyssinus and (B) D. farinae after SCIT - FAS.

Two-sided ANCOVA p-values were for comparisons between the active dose group and placebo. The bars show the LS means. The whiskers show the upper or lower limits of the $95 \% \mathrm{Cls}$.

D. pteronyssinus: Dermatophagoides pteronyssinus; D. farinae: Dermatophagoides farinae; FAS: Full analysis set; $\mathrm{n}$ : Number of patient with data; SCIT: Subcutaneous allergen immunotherapy; TU: Therapeutic unit.

\section{Subgroup analysis}

A subgroup analysis was performed for the primary end point in asthma patients planned to include in the following confirmatory Phase III trial. In this DRF patients with mild asthma without requirement of perennial ICS were included as well. Therefore, those patients have been excluded in the subgroup analysis of the primary end point. According to this subgroup analysis the biggest effect has been found for the 18,000 TU dose group for the primary end point and confirmed the result of the clinical end point (fluticasone dose reduction) chosen for the confirmatory trial as presented in Tables 2 and 5.

\section{Discussion}

According to the primary end point the change of the swelling area $6 \mathrm{~h}$ after ICT injection, 10,000 TU shows the highest statistically significant effect after SCIT for adults with HDM-allergic bronchial asthma and rhinitis. The 
Table 4. Treatment-emergent adverse events related to trial medication - safety set.

\begin{tabular}{|c|c|c|c|c|c|c|c|c|c|c|}
\hline \multirow{2}{*}{$\begin{array}{l}\text { System organ class } \\
\text { preferred term }\end{array}$} & \multicolumn{2}{|c|}{ Placebo $(n=32)$} & \multicolumn{2}{|c|}{$2000 \mathrm{TU}(\mathrm{n}=24)$} & \multicolumn{2}{|c|}{$6000 \mathrm{TU}(\mathrm{n}=31)$} & \multicolumn{2}{|c|}{$10,000 \mathrm{TU}(\mathrm{n}=28)$} & \multicolumn{2}{|c|}{18,000 TU $(n=31)$} \\
\hline & $n(\%)$ & $\mathrm{E}$ & $n(\%)$ & E & $n(\%)$ & $\mathbf{E}$ & $n(\%)$ & $\mathrm{E}$ & $n(\%)$ & $\mathrm{E}$ \\
\hline Overall & $2(6.3)$ & 2 & $4(16.7)$ & 7 & $6(19.4)$ & 13 & $4(14.3)$ & 19 & $11(35.5)$ & 30 \\
\hline $\begin{array}{l}\text { General disorders \& } \\
\text { administration site } \\
\text { conditions }\end{array}$ & 0 & - & $4(16.7)$ & 7 & $6(19.4)$ & 11 & $4(14.3)$ & 18 & $10(32.3)$ & 26 \\
\hline Injection site erythema & 0 & - & $1(4.2)$ & 1 & $3(9.7)$ & 6 & $4(14.3)$ & 13 & $3(9.7)$ & 8 \\
\hline Injection site edema & 0 & - & $1(4.2)$ & 2 & 0 & - & $1(3.6)$ & 2 & $3(9.7)$ & 5 \\
\hline Injection site pain & 0 & - & 0 & 0 & 0 & - & 0 & - & $1(3.2)$ & 1 \\
\hline Injection site papule & 0 & - & $1(4.2)$ & 1 & 0 & - & 0 & - & 0 & - \\
\hline Injection site pruritus & 0 & - & $1(4.2)$ & 1 & $1(3.2)$ & 2 & 0 & - & $2(6.5)$ & 2 \\
\hline Injection site swelling & 0 & - & $2(8.3)$ & 2 & $2(6.5)$ & 3 & 1 (3.6) & 3 & $5(16.1)$ & 10 \\
\hline Vascular disorders & 0 & - & 0 & - & 0 & - & 0 & - & $1(3.2)$ & 1 \\
\hline Hypertension & 0 & - & 0 & - & 0 & - & 0 & - & $1(3.2)$ & 1 \\
\hline Immune system disorders & $2(6.3)$ & 2 & 0 & - & $2(6.5)$ & 2 & 0 & - & $2(6.5)$ & 2 \\
\hline Hypersensitivity & $2(6.3)$ & 2 & 0 & - & $2(6.5)$ & 2 & 0 & - & $1(3.2)$ & 1 \\
\hline Anaphylactic reaction & 0 & - & 0 & - & 0 & - & 0 & - & $1(3.2)$ & 1 \\
\hline Investigations & 0 & - & 0 & - & 0 & - & 0 & - & $1(3.2)$ & 1 \\
\hline Heart rate increased & 0 & - & 0 & - & 0 & - & 0 & - & $1(3.2)$ & 1 \\
\hline Cardiac disorders & 0 & - & 0 & - & 0 & - & $1(3.6)$ & 1 & 0 & - \\
\hline Palpitations & 0 & - & 0 & - & 0 & - & $1(3.6)$ & 1 & 0 & - \\
\hline
\end{tabular}

E: Number of TEAE; N: Number of patient in the group; n: Number of patient with TEAE; TEAE: Treatment-emergent adverse event; TU: Therapeutic unit.

Table 5. Changes in intracutaneous test late-phase reactivity for the subgroup analysis population eligible for the clinical end point inhaled corticosteroid-reduction.

\begin{tabular}{|c|c|c|c|c|c|}
\hline $\begin{array}{l}\text { Intracutaneous test } \\
\text { late-phase reactivity in } \\
\mathrm{cm}^{2}\end{array}$ & Placebo $(n=15)$ & $2000 \mathrm{TU}(n=12)$ & $6000 \mathrm{TU}(\mathrm{n}=13)$ & 10,000 TU $(n=8)$ & 18,000 PNU $(n=13)$ \\
\hline LPR $\mathrm{cm}^{2}$ & -8.16 & 6.36 & 6.40 & 8.66 & 9.91 \\
\hline$p$-value & & 0.0065 & 0.0050 & 0.0047 & 0.0004 \\
\hline
\end{tabular}

10,000 TU dose was associated with the greatest reduction in the LPR swelling area compared with placebo, which was statistically significant $(\mathrm{p}<0.0001)$. The Secondary end point analyses of EPR swelling area, and an increase in morning PEF supported the result of the primary end point.

But the clinical end point, change in the minimal ICS dose required for asthma control after SCIT, is in favor of $18,000 \mathrm{TU}$. The 18,000 TU dose demonstrated the highest efficacy for ICS reduction, as shown by the percentage of patients with fluticasone dose reduction (by two steps, $69.2 \mathrm{vs} 20.0 \%$ with placebo) and the percentage of patients without need for ICS after SCIT (84.6 vs $40.0 \%$ with placebo). Clinical efficacy was supported by induction of the highest concentrations of D. pteronyssinus- and D. farinae-specific $\operatorname{IgG}_{4}$ in the 18,000 TU group compared with all other doses ( $\mathrm{p}<0.0001$ vs placebo for both antibodies). Further the subgroup analysis for the primary end point with patients of at least moderate asthma defined according to their requirement of ICS supports these findings.

Double-blind, placebo-controlled, DRF trials of SCITs have been published in a limited number over the last decade. Thus, the optimal doses are not well established [16]. The dose-dependent efficacy in the current double-blind, placebo-controlled, randomized DRF trial is comparable to previous findings in DRF trials of other SCIT preparations for HDM allergy. In one of the first HDM DRF trials, published in 1993, dose-dependent reductions in medication (such as ICS) and PEF scores were detected in adults with asthma treated with allergen extracts of $D$. pteronyssinus for 24 months [17]. In a recently published 12-month trial, dose-dependent reductions in nasal reactivity and increases in $D$. pteronyssinus-specific $\operatorname{IgG}$ and $\mathrm{IgG}_{4}$ were detected in adults with allergic rhinoconjunctivitis treated with a mixed SCIT preparation of D. pteronyssinus and D. farinae [18]. 
According to the European Medicines Agency (EMA) and the Position Paper by the European Academy of Allergy and Clinical Immunology, provocation tests and/or clinical parameters can be used as primary end points in DRF trials $[2,19]$. Evidence from various randomized, controlled trials suggest that particularly the LPR of the ICT can resemble the responses of clinical end points to AIT [20-23]. Correlation of the ICT LPR with rhinoconjunctivitis symptom scores and medication scores has been reported for grass pollen AIT [24]. The EMA encourages the simultaneous use of different end points, which may not correlate with each other, to assess the effects of treatment on asthma, a multidimensional disease [25]. It is a strength of the current trial that several different end points were explored to identify an optimal dose. It is also an advantage that a new photographic method was used to objectively measure the LPR, as the results of LPR assessments are usually subject to high inter- and intrapersonal variations [13]. However, the current trial does have potential limitations. The male:female ratios were different in the placebo versus 18,000 TU group. Sensitization to D. pteronyssinus may differ by sex [26]. However, in general, the groups were well balanced with regards to allergy-related parameters that could affect the outcomes, such as history of allergy symptoms and HDM-specific IgE concentrations. In addition, the trial was limited by sample size, which was not planned to show statistically significant differences between the different active doses for the efficacy end points. In particular, the steroid sparing effect of AIT was investigated in a subset of 63 patients who had asthma control at baseline when treated with fluticasone (i.e., 73 patients without need of fluticasone for asthma control at baseline were excluded from this analysis). The results of the current trial will utilized for the planning of a larger pivotal trial, to gather more information on the benefit-risk ratio and to find the optimal vaccine dose for the treatment of patients with asthma, rhinitis or rhinoconjunctivitis induced by HDM exposure.

The steroid sparing effect of SCIT will be used as the end point in the subsequent pivotal trial. The EMA recommends the investigation of the steroid sparing effect as a possible primary end point for Phase III studies to evaluate the efficacy of AIT in patients with allergic bronchial asthma [25]. Reduction of ICS while maintaining asthma control as the end point for AIT is based on the concept of disease control recommended by asthma guidelines $[27,28]$. The steroid sparing effect of SCIT and sublingual immunotherapy (SLIT) has been investigated in a number of double-blind, placebo-controlled trials [29-31] in adults as well as in children in an open-label trial [9].

Although the biggest efficacy for most of the end points was observed at 10,000 TU and 18,000 TU in the current study, efficacy could also be demonstrated at $6000 \mathrm{TU}$. In the $6000 \mathrm{TU}$ group versus placebo, reductions in LPR swelling area reached statistical significance $(\mathrm{p}<0.05)$, as did induction of $D$. pteronyssinus- and $D$. farinae-specific $\operatorname{IgG}\left(\mathrm{p}=0.0002\right.$ in both instances) and $\operatorname{IgG}_{4}$ concentrations $(\mathrm{p}<0.0001$ in both instances $)$ and improvements in asthma control as shown using the ACT questionnaire $(\mathrm{p}=0.0432)$. These results are comparable to efficacy outcomes in previous trials of the same SCIT using $6000 \mathrm{TU}$ as the maintenance dose, including reductions in skin and nasal reactivity in adults with rhinoconjunctivitis during a 2-year double-blind, placebo-controlled phase [32] and the steroid sparing effect in the aforementioned open-label trial following 2 years of SCIT in children with asthma [9]. These beneficial clinical effects were accompanied by the induction of $D$. pteronyssinus-specific $\operatorname{IgG}_{4}$ levels $[9,32]$.

All vaccine doses tested in this trial were well tolerated. Except for mild or moderate local reactions, which were more frequent at higher doses, all doses were comparable to placebo with respect to occurrence of trial medication-related TEAEs. Systemic reactions were rare and observed with similar incidence in the active and placebo groups. Laboratory measurements, vital signs and lung function tests did not reveal relevant differences between the different dose and placebo groups.

\section{Conclusion}

Considering all efficacy end points, including objective assessment of the ICT-induced LPR with a new photographic technique and the safety profile of all treatment groups, the HDM allergoid at the dose of $6000 \mathrm{TU}$ and above has been shown to be safe and efficacious. However, the optimal dose might be even higher. Taking into account the subgroup analysis of the patients with at least moderate asthma the 18,000 TU group showed the highest reduction in the intracutaneous LPR and ICS reduction. These efficacy results of the dose of 18,000 TU was supported by the highest serum specific antibody responses. Compared with the lower doses the 18,000 TU showed more local reactions, which were still within the normal range for SCIT. Taking into account, that patients with at least moderate asthma, are in the focus of the development program in the indication of asthma, 18,000 TU will be further investigated in a confirmatory trial to gather more information on the benefit-risk ratio of this treatment. 
Summary points

- Subcutaneous allergen immunotherapy (SCIT) with four increasing doses of a Dermatophagoides pteronyssinus allergoid was investigated in a multicenter randomized placebo-controlled double-blind clinical trial.

- The primary end point for efficacy was the change in late-phase response measured $6 \mathrm{~h}$ after intracutaneous injection of allergen assessed at baseline and after SCIT.

- Major secondary end point was the change of the minimal inhaled corticosteroid dose necessary for asthma control assessed at baseline and after SCIT.

- All four active groups showed a statistically significant reduction of the late-phase response after intracutaneous testing as compared with placebo.

- The highest dose shows the best efficacy for the inhaled corticosteroid reduction and most other end points compared with lower doses.

- Treatment was shown to be safe at all doses tested.

- The highest dose of 18,000 TU will further be investigated to treat allergic asthma induced by house dust mites.

\section{Supplementary data}

To view the supplementary data that accompany this paper please visit the journal website at: https://www.futuremedicine.com/d oi/suppl/10.2217/imt-2018-0087

Financial \& competing interests disclosure

This trial was sponsored by Allergopharma GmbH \& Co. KG, Reinbek, Germany. Data in this paper were presented at the Deutscher Allergologiekongress, Cologne, Germany 2015 and the European Academy of Allergy and Clinical Immunology Congress, Vienna, Austria 2016. The submitting agent is employee of Allergopharma. The authors have no other relevant affiliations or financial involvement with any organization or entity with a financial interest in or financial conflict with the subject matter or materials discussed in the manuscript apart from those disclosed.

M Riley at Trilogy Writing \& Consulting Ltd, Cambridge, UK, provided medical writing support on behalf of the trial sponsor, Allergopharma GmbH \& Co. KG, Reinbek, Germany.

Ethical conduct of research

The authors state that they have obtained appropriate institutional review board approval or have followed the principles outlined in the Declaration of Helsinki for all human or animal experimental investigations. In addition, for investigations involving human subjects, informed consent has been obtained from the participants involved.

\section{Open access}

This work is licensed under the Creative Commons Attribution4.0 License. To view a copy of this license, visit http://creativecomm ons.org/licenses/by/4.0/

\section{References}

Papers of special note have been highlighted as: $\bullet$ of interest

1. Thomas WR. House dust allergy and immunotherapy. Hum. Vacc. Immunother. 8, 1469-1478 (2012).

2. Pfarr O, Demoly P, Gerth van Wijk R et al. European Academy of Allergy and Clinical Immunology. Recommendations for the standardization of clinical outcomes used in allergen immunotherapy trials for allergic rhinoconjunctivitis: an EAACI position paper. Allergy 69, 854-867 (2014).

3. Jutel M, Kosowska A, Smolinska S. Allergen immunotherapy: past, present, and future. Allergy Asthma Immunol. Res. 8, 191-197 (2016).

- Recent review provides a comprehensive overview of recently developed novel allergen immunotherapy (AIT) techniques as well as further research perspectives and challenges in AIT.

4. Puttonen E, Maasch HJ, Pilström L. Studies on allergen and allergoid preparations from purified Timothy (Phleum pratense) pollen extracts. I. Physicochemical characteristics and binding to allergen-specific human IgE. Int. Arch. Allergy Appl. Immunol. 68, 1-6 (1982).

5. Puttonen E, Pilström L, Wahn U, Maasch HJ. Studies on allergen and allergoid preparations from purified Timothy (Phleum pratense) pollen extracts. II. Anaphylaxis studies in rats and histamine release from human leukocytes. Int. Arch. Allergy Appl. Immunol. 68, 7-12 (1982).

6. Wihl JA, Pilström L, Maasch HJ. Studies on allergen and allergoid preparations from purified Timothy (Phleum pratense) pollen extracts. III. Comparative investigations by skin prick tests and nasal provocation tests. Int. Arch. Allergy Appl. Immunol. 76, 162-167 (1985). 
7. Kahlert H, Grage-Griebenow E, Stüwe HAT, Cromwell O, Fiebig H. T-cell reactivity with allergoids: influence of the type of APC. J. Immunol. 165, 1807-1815 (2000).

8. Maasch HJ, Marsh DG. Standardized extracts modified allergens - allergoids. Clin. Rev. Allergy 5, 89-106 (1987).

9. Zielen S, Kardos P, Madonini E. Steroid-sparing effects with allergen-specific immunotherapy in children with asthma: a randomized controlled trial. J. Allergy Clin. Immunol. 125, 942-949 (2010).

- Pediatric trial shows treatment efficacy of one of the allergoid preparation doses investigated in current dose range finding (DRF) finding study.

10. Durham SR, Walker SM, Varga EM et al. Long-term clinical efficacy of grass-pollen immunotherapy. N. Engl. J. Med. 341, 468-475 (1999).

11. Francis JN, James LK, Paraskevopoulos G et al. Grass pollen immunotherapy: IL-10 induction and suppression of late responses precedes IgG4 inhibitory antibody activity. J. Allergy Clin. Immunol. 121, 1120-1125 (2008).

12. Martinez-Cocera C, Sastre J, Cimarra M et al. Immunotherapy with a Phleum pratense allergen extract induces an immune response to a grass-mix allergen extract. J. Investig. Allergol. Clin. Immunol. 20, 13-19 (2010).

13. Global Initiative for Asthma (GINA). Global strategy for asthma management and prevention. (2006). www.ginasthma.org

14. Rudert M, Tribanek M, Karjalainen M, Haefner D, Narkus. New objective method to measure skin test results within clinical trials. Allergy 68(Suppl. 97) 71-82 (2013).

15. Cox L, Larenas-Linnemann D, Lockey RF, Passalacqua G. Speaking the same language: the world allergy organization subcutaneous immunotherapy systemic reaction grading system. J. Allergy Clin. Immunol. 125, 569-574 (2010).

16. Calderon MA, Casale TB, Nelson HS, Demoly P. An evidence-based analysis of house dust mite allergen immunotherapy: a call for more rigorous clinical studies. J. Allergy Clin. Immunol. 132, 1322-1336 (2013).

- Postulates the conductance of better-designed clinical trials in subcutaneous immunotherapy (SCIT) involving a higher number of patients to prove the effecticacy of house dust mite AIT.

17. Haugaard L, Dahl R, Jacobsen L. A controlled dose-response study of immunotherapy with standardized, partially purified extract of house dust mite: clinical efficacy and side effects. J. Allergy Clin. Immunol. 91, 709-722 (1993).

18. Pfaar O, Nell MJ, Boot JD et al. A randomized, 5-arm dose finding study with a mite allergoid SCIT in allergic rhinoconjunctivitis patients. Allergy 71, 967-976 (2016).

19. European Medicines Agency (EMA). Guideline on the clinical development of products for specific immunotherapy for the treatment of allergic diseases (CHMP/EWP/18504/2006), (2008). www.ema.europa.eu

- Guidelines provided the basis for the design and the end point selection for the current DRF trial.

20. Bernstein IL, Li JT, Bernstein DI et al. American Academy of Allergy, Asthma and Immunology; American College of Allergy, Asthma and Immunology. Allergy diagnostic testing: an updated practice parameter. Ann. Allergy Asthma Immunol. 100, S1-S148 (2008).

21. Ferrer M, Burches E, Peláez A et al. Double-blind, placebo-controlled study of immunotherapy with Parietaria Judaica: clinical efficacy and tolerance. J. Investig. Allergol. Clin. Immunol. 15, 283-292 (2005).

22. Oldfield WL, Larche M, Kay AB. Effect of T-cell peptides derived from Fel d 1 on allergic reactions and cytokine production in patients sensitive to cats: a randomised controlled trial. Lancet 360, 47-53 (2002).

23. Parker WA Jr, Whisman BA, Apaliski SJ, Reid MJ. The relationship between late cutaneous responses and specific antibody responses with outcome of immunotherapy for seasonal allergic rhinitis. J. Allergy Clin. Immunol. 84, 667-677 (1989).

24. Lima MT, Wilson D, Pitkin L et al. Grass pollen sublingual immunotherapy for seasonal rhinoconjunctivitis: a randomized controlled trial. Clin. Exp. Allergy 32, 507-514 (2002).

25. European Medicines Agency (EMA). Note for guidance on clinical investigation of medicinal products for treatment of asthma (CHMP/EWP/2922/01 Rev.1), (2015). www.ema.europa.eu

- Guidelines provided the basis for the selection and implementation of secondary end points including inhaled corticosteroid reduction-sparing effect.

26. Goldhahn K, Bockelbrink A, Nocon M et al. Sex-specific differences in allergic sensitization to house dust mites: a meta-analysis. Ann. Allergy Asthma Immunol. 102, 487-494 (2009).

27. Global Initiative for Asthma (GINA). Global strategy for asthma management and prevention, (2014). www.ginasthma.org

28. Reddel HK, Taylor DR, Bateman ED et al. American Thoracic Society/European Respiratory Society Task Force on Asthma Control and Exacerbations. An official American Thoracic Society/European Respiratory Society statement: asthma control and exacerbations. standardizing end points for clinical asthma trials and clinical practice. Am. J. Respir. Crit. Care Med. 180, 59-99 (2009).

29. Blumberga G, Groes L, Dahl R. SQ-standardized house dust mite immunotherapy as an immunomodulatory treatment in patients with asthma. Allergy 66, 178-185 (2011).

30. Mosbech H, Deckelmann R, de Blay F et al. Standardized quality (SQ) house dust mite sublingual immunotherapy tablet (ALK) reduces inhaled corticosteroid use while maintaining asthma control: a randomized, double-blind, placebo-controlled trial. J. Allergy Clin.

Immunol. 134, 568-575 (2014). 
31. Wang L, Yin J, Fadel R, Montagut A, de Beaumont O, Devillier P. House dust mite sublingual immunotherapy is safe and appears to be effective in moderate, persistent asthma. Allergy 69, 1181-1118 (2014).

32. Dokic D, Schnittker J, Narkus A, Cromwell O, Frank E. Clinical effects of specific immunotherapy with a new house dust mite allergoid (Acaroid ${ }^{\circledR}$ ). Allergo. J. 14, 337-343 (2005). 
